\title{
DETECTION OF WATER AND/OR HYDROXYL ON ASTEROID (16) Psyche
}

\author{
Driss Takir ${ }^{1}$, Vishnu Reddy ${ }^{2}$, Juan A. Sanchez ${ }^{3}$, Michael K. Shepard ${ }^{4}$, and Joshua P. Emery ${ }^{5}$ \\ ${ }^{1}$ U.S. Geological Survey, Astrogeology Science Center, Flagstaff, AZ 86001, USA; dtakir@usgs.gov \\ ${ }^{2}$ Lunar and Planetary Laboratory, University of Arizona, Tucson, AZ 85721, USA \\ ${ }^{3}$ Planetary Science Institute, 1700 E Fort Lowell Road, Suite 106, Tucson, AZ 85719, USA \\ ${ }^{4}$ Department of Geography and Geosciences, Bloomsburg University of Pennsylvania, 400 E. Second Street, Bloomsburg, PA 17815, USA \\ 5 Earth and Planetary Science Department, Planetary Geosciences Institute, University of Tennessee, Knoxville, TN 37996, USA \\ Received 2016 August 12; revised 2016 September 21; accepted 2016 September 30; published 2016 December 28
}

\begin{abstract}
In order to search for evidence of hydration on M-type asteroid (16) Psyche, we observed this object in the $3 \mu \mathrm{m}$ spectral region using the long-wavelength cross-dispersed (LXD: 1.9-4.2 $\mu \mathrm{m}$ ) mode of the SpeX spectrograph/ imager at the NASA Infrared Telescope Facility. Our observations show that Psyche exhibits a $3 \mu \mathrm{m}$ absorption feature, attributed to water or hydroxyl. The $3 \mu \mathrm{m}$ absorption feature is consistent with the hydration features found on the surfaces of water-rich asteroids, attributed to $\mathrm{OH}$ - and/or $\mathrm{H}_{2} \mathrm{O}$-bearing phases (phyllosilicates). The detection of a $3 \mu \mathrm{m}$ hydration absorption band on Psyche suggests that this asteroid may not be a metallic core, or it could be a metallic core that has been impacted by carbonaceous material over the past 4.5 Gyr. Our results also indicate rotational spectral variations, which we suggest reflect heterogeneity in the metal/silicate ratio on the surface of Psyche.
\end{abstract}

Key words: minor planets, asteroids: general

Supporting material: data behind figure

\section{INTRODUCTION}

(16) Psyche, an M-type asteroid (Tholen 1984), is thought to be one of the most massive exposed iron metal bodies in the asteroid belt (Bell et al. 1989). Bottke et al. (2006) found that iron-meteorite parent bodies, including Psyche, are most probably formed in the terrestrial planet region. Recent polarimetric and radar estimates suggest that Psyche has an irregular shape with a diameter of $\sim 200 \mathrm{~km}$ (Lupishko 2006; Shepard et al. 2008). The high radar albedos of Psyche suggest that it is dominantly composed of metal (almost entirely $\mathrm{Fe}-$ Ni; Shepard et al. 2008; Sanchez et al. 2016). Psyche's high thermal inertia (133 or $114 \pm 40 \mathrm{~J} \mathrm{~m}^{-2} \mathrm{~S}^{-0.5} \mathrm{~K}^{-1}$; Matter et al. 2013) and density $\left(6.98 \pm 0.58 \mathrm{~g} \mathrm{~cm}^{-3}\right.$; Kuzmanoski \& Koračević 2002) provide more evidence that this asteroid has a metal-rich surface.

M-type asteroids are part of the spectroscopic X-complex taxonomy with featureless and moderately red spectra in the visible and near-infrared (NIR) region $(0.3-2.5 \mu \mathrm{m}$; Bus \& Binzel 2002; DeMeo et al. 2009). However, Hardersen et al. (2005) and Fornasier et al. (2010) found weak 0.9 and $0.43 \mu \mathrm{m}$ absorption features on several M-asteroids, including Psyche, indicating the presence of anhydrous (e.g., orthopyroxene) and hydrous (e.g., serpentine) minerals on their surfaces. An absorption feature at $1.9 \mu \mathrm{m}$, possibly attributed to mafic minerals, was also detected in several M-type asteroids (e.g., Ockert-Bell et al. 2010; Hardersen et al. 2011; Neeley et al. 2014). Recent NIR observations by Sanchez et al. (2016) revealed rotational spectral variations indicating a possible change in the metal/silicate ratio on the surface of Psyche.

Here we present the first comprehensive study to detect and interpret the $3 \mu \mathrm{m}$ absorption feature on Psyche as a function of rotation phase. In a companion paper (Sanchez et al. 2016), these data are combined with rotationally resolved NIR spectra and radar observations of Psyche to provide a more comprehensive view of the asteroid.

\section{METHODOLOGY}

\subsection{Observational Techniques}

Spectra of Psyche were measured using the long-wavelength cross-dispersed (LXD: 1.9-4.2 $\mu \mathrm{m}$ ) mode of the SpeX spectrograph/imager at the NASA Infrared Telescope Facility (IRTF). LXD data were obtained during two different nights in 2015 December. Observational circumstances are presented in Table 1. SpeX has two detectors: a Teledyne $2048 \times 2048$ Hawaii-2RG array for the spectrograph and a science grade $1024 \times 1024$ Aladdin $3 \mathrm{InSb}$ array that images the slit (Rayner et al. 2003). We were able to obtain K-band (1.95-2.5 $\mu \mathrm{m}$ ) and L-band $(2.85-4.1 \mu \mathrm{m})$ data simultaneously, using the LXD mode of SpeX and an $0.8 \times 15$ arcsec slit with an image scale of 0.15 arcsec pixel ${ }^{-1}$. The LXD mode has a resolving power $(R=\lambda / \Delta \lambda)$ of $\sim 1000$ and is covered in six orders, which are recorded simultaneously on the detector and stacked on the chip to get relatively broad wavelength coverage (Rayner et al. 2003). These orders were extracted separately and spliced together in the reduction process.

\subsection{Data Reduction}

To reduce Psyche's $3 \mu \mathrm{m}$ data, we used the Interactive Data Language-based spectral reduction tool Spextool (v4.0) (Cushing 2004). To remove the background sky (mostly $\mathrm{OH}$ line emission through most of the wavelength range and thermal emission from the sky and telescope longward of $\sim 2.3 \mu \mathrm{m}$ ), we subtracted spectra of Psyche and a standard star at beam position A from spectrum at beam B. We extracted spectra by summing the flux at each channel within a userdefined aperture. Asteroid spectra were shifted to sub-pixel accuracy to align with the calibration standard star spectra and then were divided by appropriate calibration star spectra at the same airmass $( \pm 0.05)$ to remove telluric water vapor absorption features. LXD spectra were smoothed and binned to lower spectral resolution in order to improve the signal-to-noise ratio. 
Table 1

Observational Circumstances

\begin{tabular}{|c|c|c|c|c|c|c|}
\hline Observation Date & Std. Star & Mid. UTC & Phase Angle $\left({ }^{\circ}\right)$ & Mag. $(V)$ & Long. $\left(^{\circ}\right)$ & Rot. Phase $\left({ }^{\circ}\right)$ \\
\hline 2015 Dec 08 (set 1) & SAO 94309 & 11:08:44 & 1.8 & 9.4 & 292 & 68 \\
\hline 2015 Dec 08 (set 2) & SAO 94309 & $12: 15: 15$ & 1.8 & 9.4 & 197 & 163 \\
\hline 2015 Dec 08 (set 3) & SAO 94309 & $13: 20: 44$ & 1.8 & 9.4 & 103 & 257 \\
\hline
\end{tabular}

Note. The columns in this table are date (UTC), the standard star used in the observations, mid UTC, phase angle, $V$-magnitude, body-centered longitude, and rotation phase.
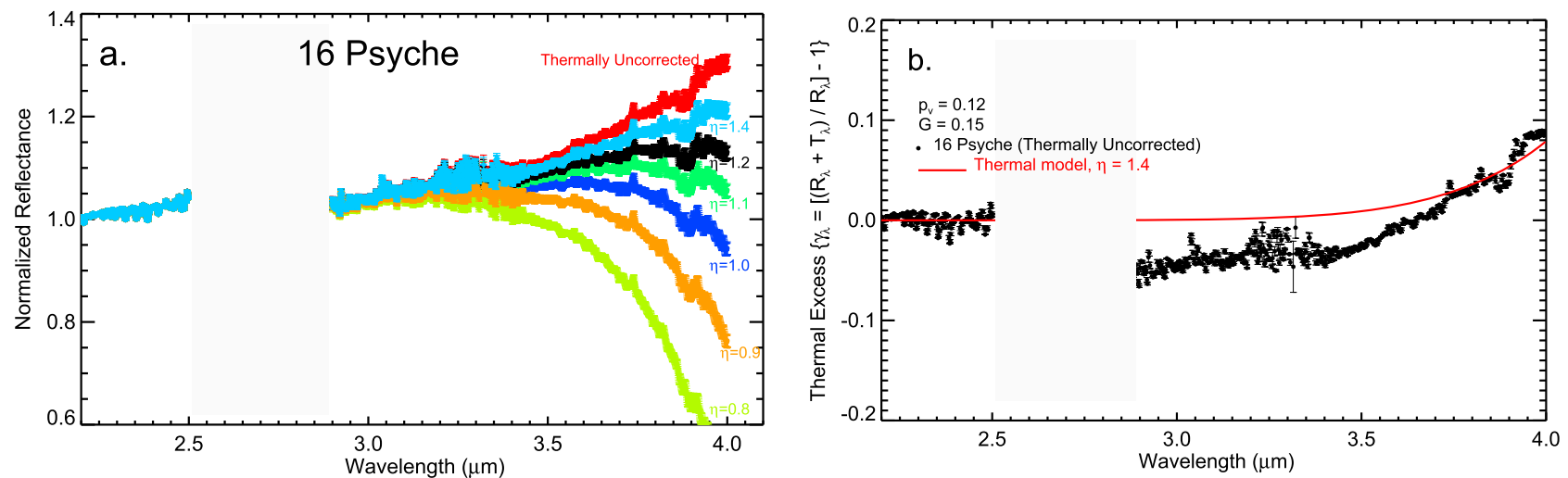

Figure 1. (a) Spectrum of asteroid Psyche, uncorrected (red) and corrected using thermal models with changing beaming parameter ( $\eta$ ) values $(0.8-1.4)$. (b) Best fit thermal model (in red with a beaming parameter of $\eta=1.4$ ) to thermally correct spectra of Psyche.

We conducted wavelength calibration at $\lambda>2.5 \mu \mathrm{m}$ using telluric absorption lines.

\subsection{Thermal Tail Removal}

We removed the thermal excess in Psyche's spectra using the methodology described in Takir \& Emery (2012) and references therein. The thermal excess $\gamma_{\lambda}$ is defined as a measure of the thermal flux found at these wavelengths by

$$
\gamma_{\lambda}=\frac{R_{\lambda}+T_{\lambda}}{R_{\lambda}}-1,
$$

where $R_{\lambda}$ is the reflected flux at a wavelength $\lambda, T_{\lambda}$ is the thermal flux at a given wavelength, and the quantity $R_{\lambda}+T_{\lambda}$ is the measured relative spectrum. To constrain Psyche's model thermal flux, we fitted the measured thermal excess with a model thermal excess. Then, we subtracted this model thermal flux from the measured relative spectrum of Psyche.

To calculate the thermal flux in the $3 \mu \mathrm{m}$ region, we used the Near-Earth Asteroid Thermal Model (Harris 1998), which is based on the Standard Thermal Model of Lebofsky et al. (1986). We obtained asteroid Psyche inputs, including heliocentric and geocentric distances, geometric albedo, and phase angle at time of observation from the Jet Propulsion Laboratory Horizon online ephemeris generator. We used the value of 0.15 as the default value for the slope parameter $G$ (Bowell et al. 1989 ). This $G$ value is also consistent with the published value of $G\left(0.12_{-0.03}^{+0.04}\right)$ for Psyche in the Asteroid Absolute Magnitude and Slope Catalog (Muinonen et al. 2010; Oszkiewicz et al. 2011). Figure 1(a) shows the effect of changing the beaming parameter thermal corrections in Psyche's spectra. Figure 1(b) shows the chosen thermal model $\left(\eta=1.4, p_{\mathrm{v}}=0.12\right)$ to thermally correct spectra of Psyche.
Table 2

$3 \mu \mathrm{m}$ Band Analysis Showing Spectra of Psyche to Exhibit an Absorption Feature around $3 \mu \mathrm{m}$ Spectral Region

\begin{tabular}{lcc}
\hline \hline Observation Date & Mid. UTC & $3 \mu \mathrm{m}$ Band Depth $(\%)$ \\
\hline 2015 Dec 08 (set 1$)$ & $11: 08: 44$ & $2.84 \pm 1.37$ \\
2015 Dec 08 (set 2) & $12: 15: 15$ & $3.04 \pm 1.21$ \\
2015 Dec 08 (set 3) & $13: 20: 44$ & $3.26 \pm 1.29$ \\
2015 Dec 09 (set 4) & $14: 14: 40$ & $2.72 \pm 1.81$ \\
\hline
\end{tabular}

Note. A $1 \%$ uncertainty was added to our calculated $3 \mu \mathrm{m}$ band depth uncertainty as described in Section 2.5.

\subsection{Continuum Slope Choice}

The thermal excess removal technique described in Section 2.3 requires an estimate of the reflected continuum in the thermal regime. The nominal approach is to fit a regression line to the K-band portion of the spectrum and extend it through the L-band portion. However, the slope of this reflectance continuum directly affects the band depth estimate. This dependence is not because of the thermal correction; as Figure 1 shows, the thermal correction is minimal in the 2.9-3.0 $\mu \mathrm{m}$ region, regardless of thermal model parameters. However, the slope of the reflectance continuum sets the reflectance from which the band depth is measured.

In order to assess the contribution of continuum slope uncertainty to our overall band depth uncertainty, we varied the slope of the regression line and shifted it vertically, then redid the thermal correction described in Section 2.3. In all cases, varying the slope by $0.02 \mu \mathrm{m}^{-1}$ up or down under- or overcorrects the thermal tail a noticeable amount, so we consider that to be the reasonably possible range for the reflectance continuum. Those changes in slope change the band depth by $\sim 1 \%$. Therefore, a $1 \%$ uncertainty was added to the uncertainty 


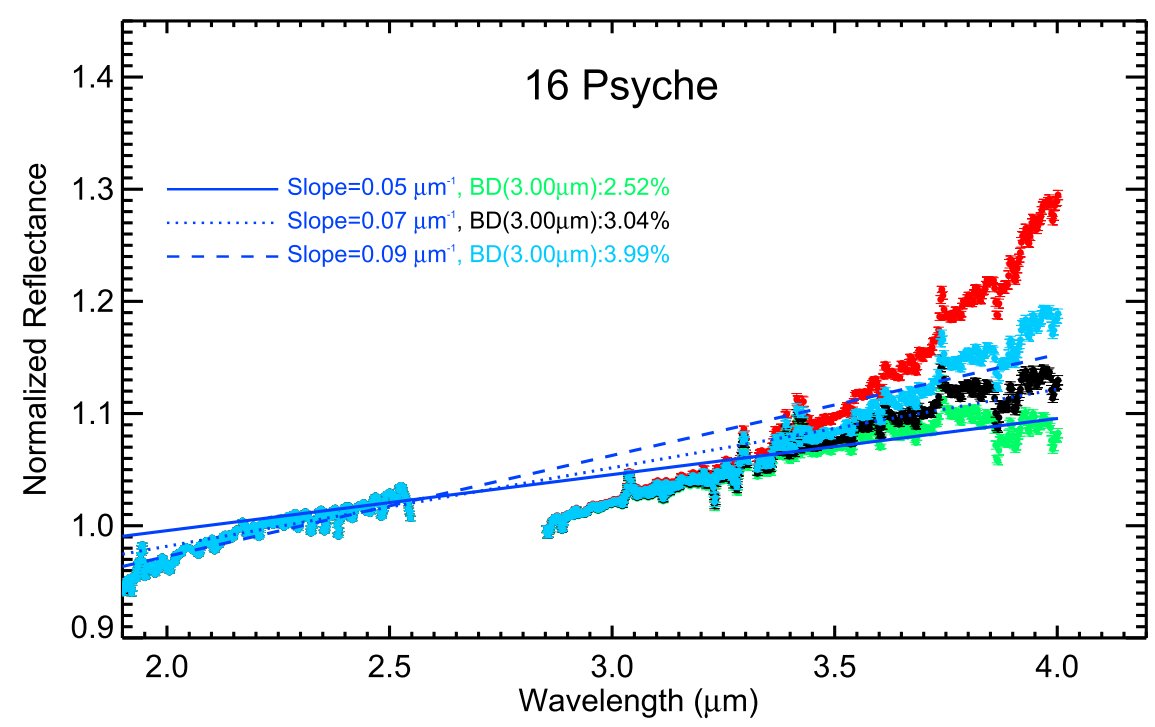

Figure 2. Illustration of the effect of the continuum choice on the measured band depth. The uncorrected spectrum of set 2 is shown in red. The other three spectra are the optimally corrected spectra (following the procedure described in Section 2.3) for each of three choices of continuum slope, as labeled on the figure. The slope of $0.07 \mu \mathrm{m}^{-1}$ is the best fit to the data. For slopes steeper than $0.09 \mu \mathrm{m}^{-1}$, all thermal models under-correct the thermal excess, and for slopes shallower than $0.05 \mu \mathrm{m}{ }^{-1}$, all thermal models over-correct the thermal excess.

of our calculated $3 \mu \mathrm{m}$ band depth (Table 2). An example of the thermal excess removal for different reflectance continuum slopes is shown in Figure 2 for data set 2 (2015 December 8).

\section{5. $3 \mu \mathrm{m}$ Band Depth and Uncertainty}

We calculated the band depth, $D_{3.00}$, at $3 \mu \mathrm{m}$ relative to the continuum (the linear regression line across the K-band) using the following equation:

$$
D_{3.00}=\frac{R_{c}-R_{3.00}}{R_{c}}
$$

where $R_{3.00}$ represents the reflectance at $3 \mu \mathrm{m}$, and $R_{c}$ represents the reflectance of the continuum at the same wavelength as $R_{3.00}$.

The uncertainty in $D_{\lambda}$ (the criterion is a $2 \sigma$ detection) was computed using the following equations (Taylor 1982, p. 55):

$$
\delta D_{3.00}=D_{3.00} * \sqrt{\left(\frac{\delta R_{1}}{R_{1}}\right)^{2}+\left(\frac{\delta R_{c}}{R_{c}}\right)^{2}},
$$

where $\delta R_{1}=\sqrt{\left(\delta R_{c}\right)^{2}+\left(\delta R_{3.00}\right)^{2}}$.

We also added a $1 \%$ uncertainty in quadrature to the computed uncertainties $\left(\sqrt{\delta D_{3.00}^{2}+0.01^{2}}\right)$ to account for the uncertainty in the L-band continuum slope in all spectra.

\section{RESULTS}

Figures 3(a)-(d) show the LXD spectra of asteroid (16) Psyche, which were obtained during two nights in 2015 December. All these spectra show absorption features around $3 \mu \mathrm{m}$ region. Table 2 shows our $3 \mu \mathrm{m}$ band analysis.

LXD spectra of Psyche corresponding to four different rotation phases are shown in Figure 4. These data have been combined with the average NIR spectrum of Psyche obtained by Sanchez et al. (2016) with the IRTF, so we cover the full wavelength range from $\sim 0.7$ to $4.2 \mu \mathrm{m}$. For each rotation phase, shape models of Psyche are also shown. The threedimensional shape models of Psyche were created with the SHAPE software package (Magri et al. 2007), using data obtained by Sanchez et al. (2016), which include Arecibo delay-Doppler radar observations and adaptive optics images from Keck and Magellan. A detailed description of the procedure can be found in Sanchez et al. (2016).

\section{DISCUSSION}

Our $3 \mu \mathrm{m}$ observational results revealed that Psyche may not be as featureless as once thought in the $3 \mu \mathrm{m}$ spectral region. Because asteroid spectra measured with ground-based telescopes are affected by strong absorptions by water vapor in Earth's atmosphere, our analysis of Psyche LXD spectra is restricted to the $2.8-3.3 \mu \mathrm{m}$ spectral range and the shape of the $3 \mu \mathrm{m}$ feature.

The LXD observations have revealed that Psyche exhibits a $3 \mu \mathrm{m}$ hydration absorption feature, similar to the hydration features observed on some water-rich asteroids (e.g., Takir \& Emery 2012), attributed to $\mathrm{OH}-$ and/or $\mathrm{H}_{2} \mathrm{O}$-bearing phases (e.g., Takir et al. 2013). The $3 \mu \mathrm{m}$ band shape in Psyche's spectrum is also consistent with the $3 \mu \mathrm{m}$ band found in spectra of the Moon (Clark 2009; Pieters et al. 2009; Sunshine et al. 2009), attributed to adsorbed $\mathrm{H}_{2} \mathrm{O} / \mathrm{OH}$.

Figure 5 shows there are some similarities between spectra of Psyche and the CI carbonaceous chondrite Ivuna (measured under asteroid-like conditions) in that they both have the same sharp $3 \mu \mathrm{m}$ band shape (Takir et al. 2013). This sharp shape was also found in many outer Main Belt and hydrated asteroids (Takir \& Emery 2012; Takir et al. 2015a), attributed to phyllosilicates (Figure 5). Figure 5 also shows that both spectra of Ivuna and the T-type asteroid (308) Polyxo have a similar $3 \mu \mathrm{m}$ band shape to the spectrum of Psyche with a deeper band depth (more abundance of hydrated minerals).

Rivkin et al. (2000) did not report a $3 \mu \mathrm{m}$ feature in Psyche. However, given that the uncertainties in the Rivkin et al. spectrophotometry are larger than the $\sim 3 \%$ feature observed in this work, our results are still consistent with those of Rivkin et al.

In just one set, set 1 (Figure 3(a)), the $3 \mu \mathrm{m}$ feature in Psyche is superimposed on a broader absorption from $\sim 3.25$ to $3.73 \mu \mathrm{m}$, similarly to the feature observed on Ceres (Lebofsky 

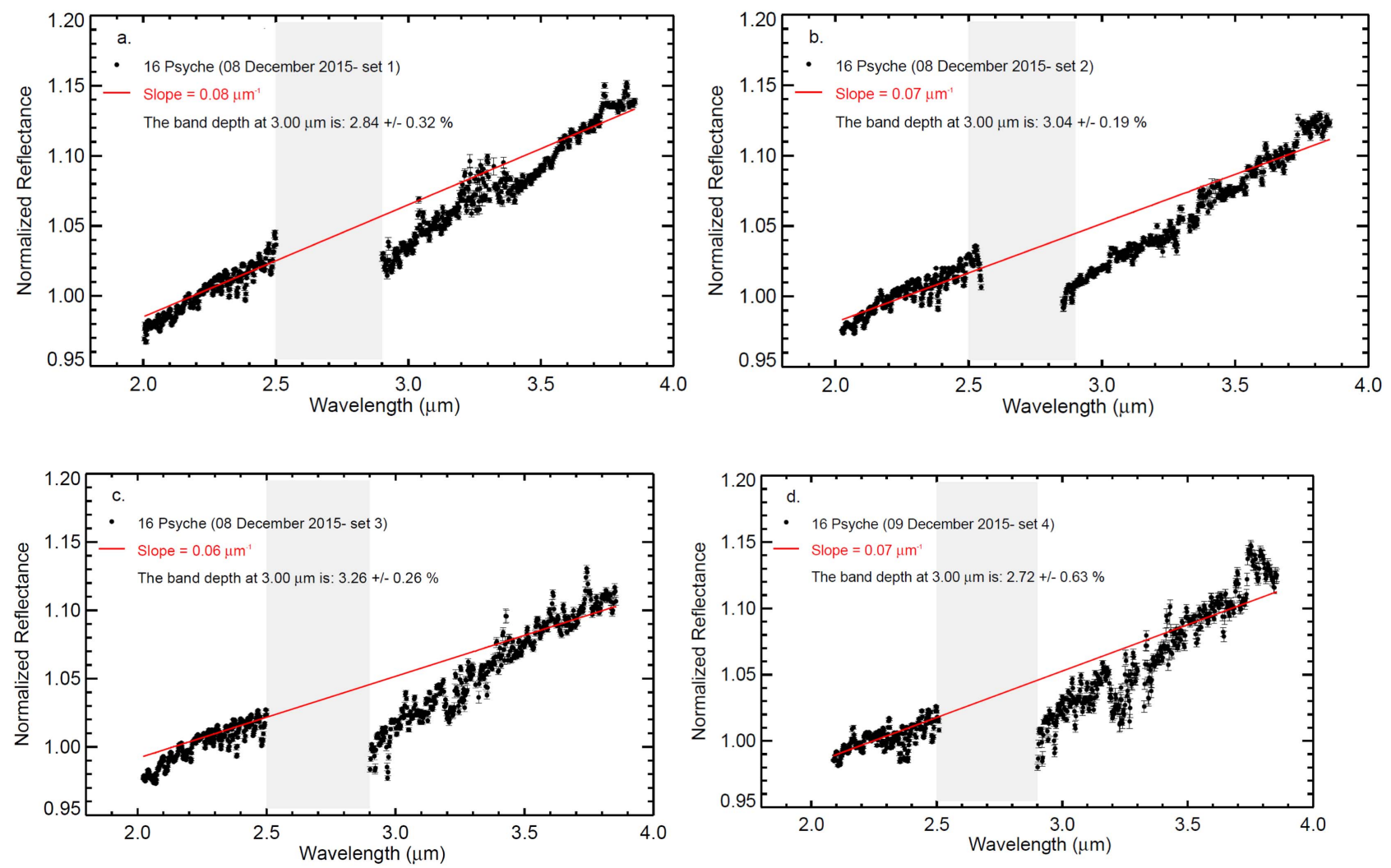

Figure 3. (a)-(d). LXD spectra of asteroid (16) Psyche obtained during two nights in 2015 December. These spectra show $3 \mu \mathrm{m}$ absorption features. The gray bars on each plot mark wavelengths of strong absorption by water vapor in Earth's atmosphere. The band depths were calculated using the methods described in Sections 2.4 and 2.5. The data used to create this figure are available.

1978; Jones et al. 1990) and on the Ceres-like group (Takir \& Emery 2012; Takir et al. 2015b). Milliken \& Rivkin (2009) attributed these absorption features on Ceres to $\mathrm{OH}$ or $\mathrm{H}_{2} \mathrm{O}-$ bearing phases. We only see this feature in set 1 (2015 December 08), which corresponds to a body-centered longitude of $292^{\circ}$, where a large crater (longitude $300^{\circ}$ ) of $85 \pm 20 \mathrm{~km}$ in diameter is located (Sanchez et al. 2016). This impact crater on Psyche may have been a product of collisions with carbonaceous chondrite projectiles, forming a scattering of carbonaceous ejecta on its surface. All spectra show features around $3.9 \mu \mathrm{m}$, which is difficult to interpret because of telluric absorptions (e.g., $\mathrm{NO}_{2}$ ) in this spectral region.

Evidence for the $3 \mu \mathrm{m}$ band was detected in spectra of many M-type asteroids, attributed to $\mathrm{OH}$ or $\mathrm{H}_{2} \mathrm{O}$-bearing phases (e.g., Jones et al. 1990; Rivkin et al. 2000; Landsman et al. 2015). Gaffey et al. (2002) and references therein proposed a number of plausible alternative interpretations for the presence of the $3 \mu \mathrm{m}$ band on $\mathrm{M}$-asteroids. These interpretations include the presence of anhydrous silicates containing structural $\mathrm{OH}$, the presence of fluid inclusions, the presence of xenolithic hydrous meteorite components on asteroid surfaces from impacts (as those seen on Vesta by Reddy et al. 2012), solar windimplanted $\mathrm{H}$, or the presence of troilite.

Rivkin et al. (2000) found that M-asteroids with diameters larger than $65 \mathrm{~km}$ are likely to be hydrated and M-asteroids with diameters smaller than $65 \mathrm{~km}$ are likely to be anhydrous. The authors suggested that hydrated M-asteroids are likely to have a mineralogy similar to hydrated enstatite chondrites or high-albedo carbonaceous chondrites. In our observations we found Psyche, a large M-asteroid with a diameter of $\sim 200 \mathrm{~km}$, to be hydrated, in agreement with the work of Rivkin et al. (2000). Our observations of Psyche are also consistent with the findings of Davis et al. (1998) who suggested that large M-asteroids, like Psyche, should not be expected to be exposed metallic cores. The authors suggested that Psyche has been shattered by impacts but is not a catastrophically disrupted metallic core (e.g., Asphaug et al. 2006).

\section{SUMMARY}

We observed asteroid (16) Psyche in the $3 \mu \mathrm{m}$ band during two nights in 2015 December, using the (LXD: 1.9-4.2 $\mu \mathrm{m}$ ) mode of the SpeX spectrograph/imager at the NASA IRTF. The LXD data were combined with the average NIR spectra and shape model of Psyche for deeper understanding of the asteroid. The observations of Psyche revealed that its spectra exhibit a $3 \mu \mathrm{m}$ absorption feature, possibly due to water or hydroxyl. The $3 \mu \mathrm{m}$ band shape in Psyche's spectra was found to be consistent with the hydration features observed on some water-rich asteroids and carbonaceous chondrite meteorites, attributed to $\mathrm{OH}-$ and/or $\mathrm{H}_{2} \mathrm{O}$-bearing minerals. The detection of a $3 \mu \mathrm{m}$ absorption band suggests that Psyche may not be a metallic core, or it could be a metallic core that has been impacted by carbonaceous material over the past $4.5 \mathrm{Gyr}$. Our results also indicate rotational spectral variations, which we suggest reflect heterogeneity in the metal/silicate ratio on the surface of Psyche. 

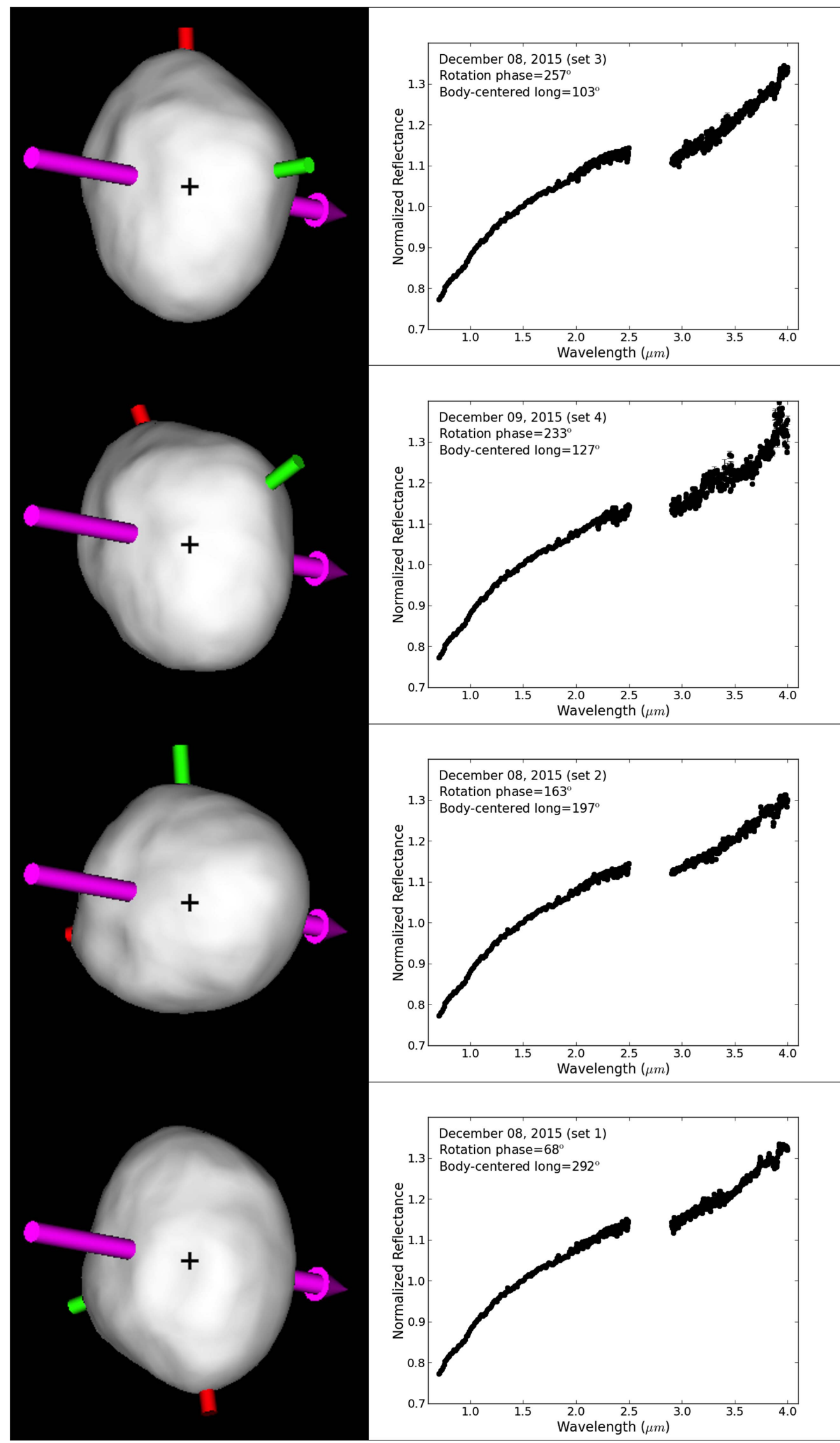

Figure 4. LXD spectra of (16) Psyche corresponding to four different rotation phases. These data have been combined with the average NIR spectrum of Psyche obtained by Sanchez et al. (2016). All spectra are normalized to unity at $1.5 \mu \mathrm{m}$. For each rotation phase, three-dimensional shape models of Psyche are also shown. The axes on the shape models correspond to longitude $0^{\circ}$ (red) and longitude $90^{\circ}$ (green). 


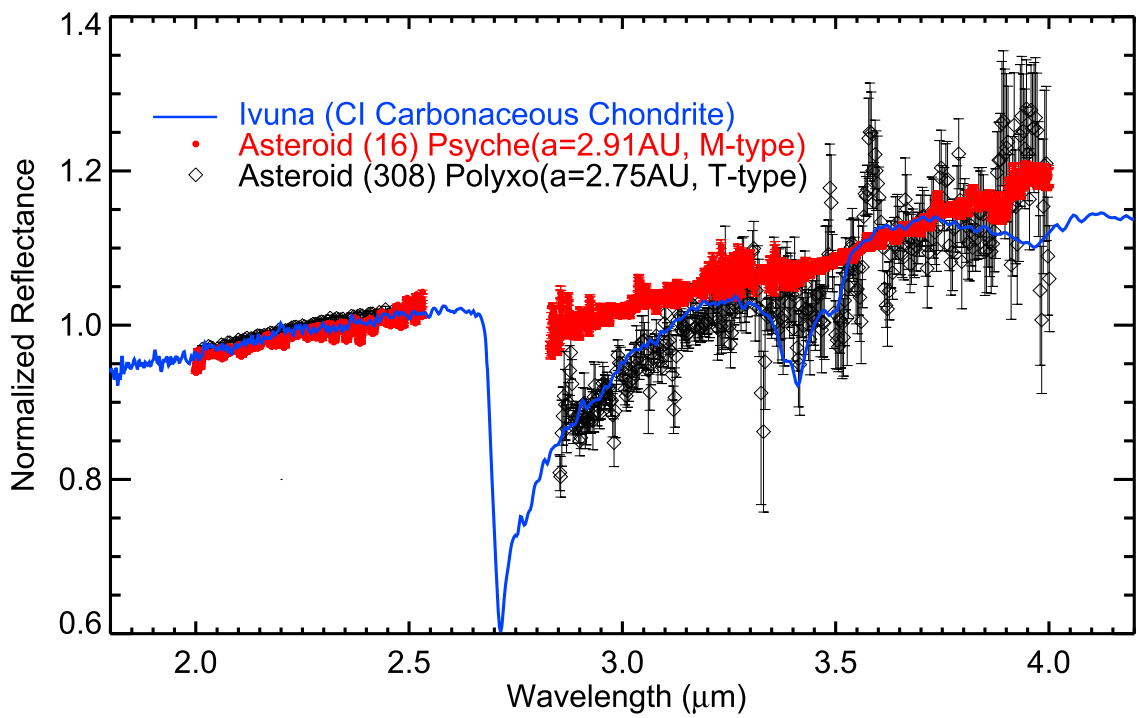

Figure 5. Psyche's spectrum is also found to be similar to the spectrum of the CI carbonaceous chondrite meteorite Ivuna (Takir et al. 2013) and T-type asteroid (308) Polyxo $(a=2.75$ au; Takir \& Emery 2012; Takir et al. 2015a). Both spectra of Ivuna and Polyxo have a similar $3 \mu \mathrm{m}$ band shape to the spectrum of Psyche with a deeper band depth (more abundance of hydrated minerals).

We wish to thank NASA IRTF staff for their assistance with asteroid observations. NASA IRTF is operated by the University of Hawai'i under contract NNH14CK55B with NASA. We are grateful for the thoughtful and excellent review by an anonymous reviewer. D.T.'s contribution to this work was funded by Eugene M. Shoemaker Postdoctoral Fellowship (NASA Planetary Geology and Geophysics Program). Work by V.R. and J.A.S. was funded by NASA Planetary Geology and Geophysics grants NNX14AN05G and NNX14AN35G. J.P. E.'s contribution to this work was funded by NASA Solar System Observing grant NNX16AE91G.

\section{REFERENCES}

Asphaug, E., Agnor, C. B., \& Quentin, W. 2006, Natur, 439, 155

Bell, J. F., Davis, D. R., Hartmann, W. K., \& Gaffey, M. J. 1989, in Asteroids

II, Asteroids - The big picture, ed. R. P. Binzel, T. Gehrels, \& M. S. Matthews (Tucson, AZ: Univ. Arizona Press), 921

Bottke, W. F., Nesvorny, D., Grimm, R. E., Morbidelli, A., \& O’Brien, D. P. 2006, Natur, 439, 821

Bowell, E., Hapke, B., Domingue, D., et al. 1989, in Asteroids II, ed. R. P. Binzel, T. Gehrels, \& M. S. Matthews (Tucson, AZ: Univ. Arizona Press), 524

Bus, S. I., \& Binzel, R. P. 2002, Icar, 158, 146

Clark, R. N. 2009, Sci, 326, 562

Cushing, M. C. 2004, PASP, 116, 362

Davis, D. R., Farinella, P., \& Marzari, F. 1998, Icar, 137, 140

DeMeo, F. E., Binzel, R. P., Slivan, S. M., \& Bus, S. J. 2009, Icar, 202, 160

Fornasier, S., Clark, B. E., Dotto, E., et al. 2010, Icar, 210, 655

Gaffey, M. J., Cloutis, E. A., Kelley, M. S., \& Reed, K. L. 2002, in Asteroids III, Mineralogy of Asteroids, ed. W. F. Bottke et al. (Tucson, AZ: Univ. Arizona Press), 183
Hardersen, P. S., Cloutis, E. A., Reddy, V., Mothe-Dinz, T., \& Emery, J. P. 2011, M\&PS, 46, 1910

Hardersen, P. S., Gaffey, M. J., \& Abell, P. A. 2005, Icar, 175, 141

Harris, A. W. 1998, Icar, 131, 291

Jones, T., Lebofsky, L., Lewis, J., \& Marley, M. 1990, Icar, 88, 172

Kuzmanoski, M., \& Koračević, A. 2002, A\&A, 395, L17

Landsman, Z. A., Campins, H., Pinilla-Alonso, N., Hanus, J., \& Lorenzi, V. 2015, Icar, 252, 186

Lebofsky, L. A. 1978, MNRAS, 182, 17

Lebofsky, L. A., Sykes, M. V., Tedesco, E. F., et al. 1986, Icar, 68, 239

Lupishko, D. F. 2006, SoSyR, 40, 214

Magri, C., Ostro, S. J., Scheeres, D. J., et al. 2007, Icar, 186, 152

Matter, A., Delbo, M., Carry, B., \& Ligori, S. 2013, Icar, 226, 419

Milliken, R. E., \& Rivkin, A. 2009, NatGe, 2, 258

Muinonen, K., Belskaya, I. N., Cellino, A., et al. 2010, Icar, 209, 542

Neeley, J. R., Clark, B. E., Ockert-Bell, M. E., et al. 2014, Icar, 238, 37

Ockert-Bell, M. E., Clark, B. E., Shepard, M. K., et al. 2010, Icar, 210, 674

Oszkiewicz, D. A., Muinonen, K., Bowell, E., et al. 2011, JQSRT, 112, 1919

Pieters, C. M., Goswami, J. N., Clark, R. N., et al. 2009, Sci, 326, 568

Rayner, J. T., Toomey, D. W., Onaka, P. M., et al. 2003, PASP, 155, 362

Reddy, V., Le Corre, L., O'Brien, D. P., et al. 2012, Icar, 221, 544

Rivkin, A. S., Howell, E. S., Lebofsky, L. A., Clark, B. E., \& Britt, D. T. 2000, Icar, 145, 351

Sanchez, J. A., Reddy, V., Shepard, M. K., Thomas, C., \& Cloutis, E. 2016, AAS/DPS Meeting 48 Abstract, \#325.20

Shepard, M. K., Clark, B. E., Nolan, M. C., et al. 2008, Icar, 195, 184

Sunshine, J. M., Farnham, T. L., Feaga, L. M., et al. 2009, Sci, 326, 565

Takir, D., \& Emery, J. P. 2012, Icar, 219, 641

Takir, D., Emery, J. P., McSween, H. Y., et al. 2013, M\&PS, 48, 1618

Takir, D., Emery, J. P., \& McSween, H. Y. 2015a, Icar, 257, 185

Takir, D., Reddy, V., Sanchez, J. A., et al. 2015b, ApJL, 804, L13

Taylor, J. R. 1982, An Introduction to Error Analysis (Mill Valley, CA: Univ. Science Books)

Tholen, D. J. 1984, PhD dissertation, Univ. Arizona 